\title{
Investigation of Exogenous Shocks in Complex Supply Networks - A Modular Petri Net approach
}

by

Gilbert Fridgen, Christian Stepanek, Thomas Wolf 


\section{Investigation of Exogenous Shocks in Complex Supply Networks - A Modular Petri Net Approach}

Fridgen, Gilbert ${ }^{\mathrm{a}}$

FIM Research Center, University of Bayreuth, Germany

Stepanek, Christian ${ }^{\mathrm{b}}$

Institute for Material Resource Management, University of Augsburg, Germany

Wolf, Thomas ${ }^{\mathrm{c}^{*}}$

FIM Research Center, University of Augsburg, Germany

${ }^{a}$ Research Center Finance \& Information Management, University of Bayreuth, Friedrich-von-Schiller-Str. 2a, 95444 Bayreuth, Germany; ${ }^{b}$ Institute for Material Resource Management, University of Augsburg, Universitaetsstrasse 2 86135 Augsburg, Germany; 'Research Center Finance \& Information Management, University of Augsburg, Universitaetsstrasse 12, 86159 Augsburg, Germany; 


\section{Investigation of Exogenous Shocks in Complex Supply Networks - A Modular Petri Net Approach}

Global supply networks that can be considered as interlaced supply chains are shaping existing economic structures. These supply networks are creating a high level of complexity. Simultaneously, the perceived number of exogenous shocks such as natural disasters is increasing. These exogenous shocks can directly or indirectly impact the participating companies of a supply network, which can also threaten the network as a whole. However, the complexity and opacity of today's supply networks inhibits an accurate prediction and quantification of such impacts. Therefore, companies are unable to develop adequate safeguards, while existing mechanisms are insufficient. The objective of this study is to model, analyze, and quantify the impacts of exogenous shocks on supply networks. Therefore, we use a Petri Net-based approach, which enables a simulation of different supply network constellations, to assess the vulnerability to exogenous shocks. Furthermore, we include a detailed description of modeling and evaluation of the presented method. For an exemplary supply network, we simulate different intensities of an exogenous shock combined with different safety stocks of the entities. Statistical tests are conducted to verify the results. We thereby illustrate the results that could be yielded from a real-world application.

Keywords: word; supply chain, supply network, Petri Net, exogenous shock 